\title{
Klinische Forschung braucht gutgeschultes Personal
}

\author{
Das Aus- und Weiterbildungskonzept der Clinical Trial Unit am Universitätsspital \\ Basel soll zur kontinuierlichen Steigerung von Kapazität und Qualität im Bereich \\ patientenorientierter Forschung beitragen.
}

Barbara C. Peters ${ }^{a}$, Christiane Pauli-Magnus ${ }^{b}$

a Dr. rer. nat., Leiterin Ressort Aus- und Weiterbildung, Clinical Trial Unit Basel

b Prof. Dr. med., Leiterin Clinical Trial Unit Basel

Korrespondenz:

Barbara C. Peters

Clinical Trial Unit

Universitätsspital Basel

Schanzenstrasse 55

CH-4031 Basel

petersb[at]uhbs.ch

www.clinicaltrialunit.ch
Die klinische Forschung unterliegt auf gesetzlich/ regulatorischem Gebiet sowie in Bezug auf die Entwicklung neuer und neuester Therapien einer grossen Dynamik. Voraussetzung für qualitativ hochstehende und kosteneffiziente Forschung ist dabei adäquat geschultes Personal, das die verschiedenen Aspekte der Studienplanung, -durchführung und -auswertung kompetent abdecken kann. Die Clinical Trial Unit (CTU) Basel, ein multidisziplinäres Kompetenzzentrum für klinische Forschung am Universitätsspital Basel, begegnet diesem Umstand mit einem umfangreichen Kursangebot für verschiedene in der patientenorientierten klinischen Forschung tätige Berufsgruppen.

\section{Kurse für Prüfärzte und Studienleiter}

Das Schulungsangebot der Clinical Trial Unit (CTU) Basel [1] umfasst Basis- und Aufbaukurse sowohl für Prüfärzte und Studienleiter als auch für nicht-ärztliches Studienpersonal aus der akademischen oder industriellen klinischen Forschung (Abb. 1). Zu den Basiskursen zählen drei vom Schweizerischen Heilmittelinstitut Swissmedic anerkannte Kurse [2]. Der Clinical Investigator Course ist ein allgemeiner dreitägiger Einführungskurs, der neben den ethischen und rechtlichen Grundlagen der klinischen Forschung [3-6] die wichtigsten Aspekte der methodischen und statistischen Studienplanung, der Prüfplanentwicklung und der praktischen Studiendurchführung behandelt. Viel Wert wird dabei auf das Bearbeiten von praktischen Beispielen und die Inter-

\section{Personnel bien formé pour la recherche clinique}

La recherche clinique est soumise à une forte dynamique aussi bien d'un point de vue légal / régulateur que du point de vue du développement de nouveaux traitements. Pour que la recherche puisse être de qualité élevée et rentable en termes de coûts, elle doit pouvoir compter sur du personnel au bénéfice d'une formation adéquate, à même de prendre en charge avec compétence les différentes tâches qui lui sont dévolues, notamment pour planifier, dispenser et évaluer l'enseignement. Le centre de compétences multidisciplinaires dédié à la recherche clinique (Clinical Trial Unit ou CTU) de I'hôpital universitaire de Bâle répond à ce constat par une offre importante de cours destinés à différents groupes professionnels actifs dans la recherche clinique axée sur le patient.

dulen absolviert werden. Ergänzend dazu wurden die Basiskurse «GCP in der Pädiatrie» und «Klinisch-chirurgische Forschung» entwickelt, die auf die besonderen ethischen Herausforderungen sowie typische Fra-

\section{Auf vielfachen Wunsch wird ein Aufbaukurs «Advanced Statistics» für eine vertiefte Schulung in statistischer Datenanalyse im Bereich klinische Forschung angeboten.}

aktion mit den Kursteilnehmern gelegt. Korrektes Verfassen eines Studienprotokolls und statistische Studienplanung sind weitere Themen. Der Clinical Investigator Course findet zwei Mal jährlich statt und kann komplett (3 Tage, 3 Module) oder in Einzelmo- gestellungen und Studiensettings in diesen Fachgebieten zugeschnitten sind.

Mit dem Aufbaukurs «Advanced Statistics» kommt die CTU Basel dem vielfachen Wunsch nach einer weiterführenden, vertieften Schulung in statistischer 


\section{Abbildung 1}

Aus- und Weiterbildungskonzept der CTU Basel: Basis- und Aufbaukurse für Studienleiter und Prüfärzte sowie für nicht-ärztliches Studienpersonal. (Swissmedic $=$ Schweizerisches Heilmittelinstitut; SwAPP = Swiss Assosiation for Pharmaceutical Professionals; SGPM = Schweizerische Gesellschaft für Pharmazeutische Medizin).

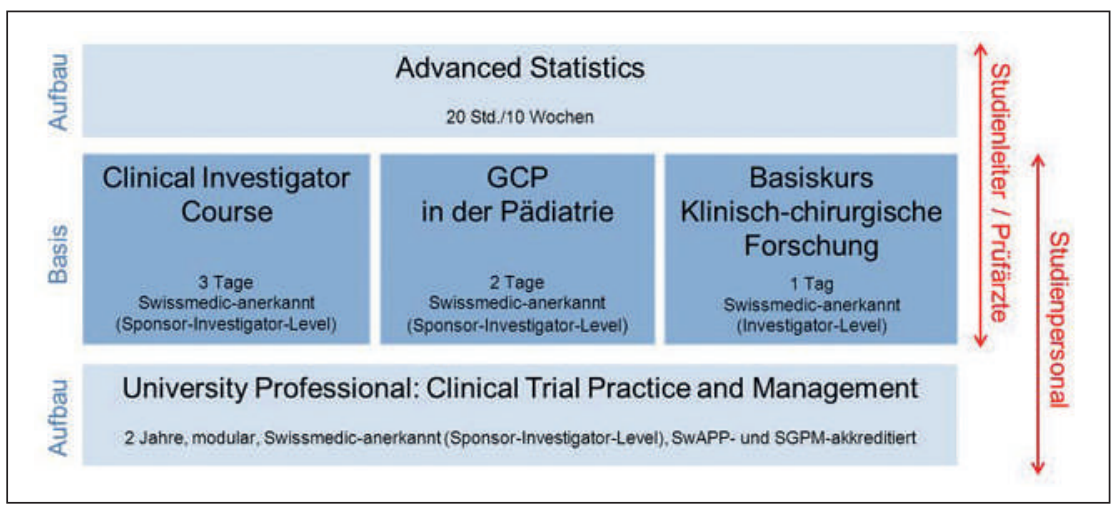

Datenanalyse speziell im Bereich klinische Forschung nach. Zielpublikum sind primär Ärzte und Studienleiter, die ihre in den Basiskursen erworbenen Kenntnisse im Bereich der Statistik und in der Verarbeitung von Daten aus klinischen Studien weiter vertiefen wollen.

\section{Lehrgang für nicht-ärztliches Studienpersonal}

Neben dem ärztlichen Personal, das für die Planung und Durchführung klinischer Studien verantwortlich zeichnet, gibt es die heterogene Berufsgruppe des nicht-ärztlichen Studienpersonals, das in den operativen Belangen der Studiendurchführung meist eine tragende Rolle spielt. Gemeint sind Personen, die unter Berufsbezeichnungen wie Study Nurse, Studienkoordinator, Clinical Trial/Research Assistant oder Clinical Monitor an den akademischen Forschungsinstitutionen, in der pharmazeutischen und BiotechIndustrie sowie bei Auftragsforschungsinstituten (Clinical Research Institutes, CROs) tätig sind.

Für diese Berufsgruppe und solche, die in diesen Bereich einsteigen wollen, gibt es mit dem universitären Lehrgang «University Professional: Clinical Trial Practice and Management» erstmals eine umfassende und vertiefende Ausbildung. Inhaltlich gibt der Lehrgang einen ausführlichen Überblick über den Entwicklungsprozess von Arzneimitteln, Medizinprodukten und Advanced Therapies, stellt ethische und rechtliche Aspekte der klinischen Forschung [3-6] detailliert vor und behandelt alle Schritte der praktischen Durchführung von klinischen Studien ausführlich und mit viel Raum für Übungen und eigenes Erfahren und Erarbeiten.

Der zweijährige Lehrgang ist berufsbegleitend strukturiert. Er besteht aus sieben Modulen, mit Lehreinheiten von 3-4 Tagen. Das dort vermittelte theoretische Wissen wird mit Einblicken in den Berufsalltag komplementiert, indem Kursteilnehmer einwöchige Praktika an Abteilungen und Institutionen absolvieren, die in verschiedene Aspekte der klinischen Forschung involviert sind. Kommunikationstraining und Förderung von selbstständigem Lösen von Aufgaben stärken persönliche Kompetenzen.

\section{Auch über Neuerungen stets informiert sein}

Gezielte und strukturierte Personalschulungen sind ein unerlässliches Instrument zur Qualitätssicherung und -steigerung. Um den steten Neuerungen und Weiterentwicklungen im Bereich der klinischen Forschung auf diesem Gebiet Rechnung zu tragen, wird die CTU Basel im kommenden Jahr aktuelle Thematiken wie das neue Humanforschungsgesetz [7] oder die Entwicklung Molekularer Therapien in einem geeigneten Weiterbildungsformat erarbeiten. Auch ein für die Aufgabenbereiche der Study Nurses zugeschnittener Einführungskurs befindet sich derzeit in Ausarbeitung.

\section{Referenzen}

1 Homepage CTU Basel: www.clinicaltrialunit.ch

2 Swissmedic. Anforderungen an die Ausbildung von Co-Prüfern, Hauptprüfern und Sponsor-Prüfern im Rahmen von klinischen Versuchen mit Heilmitteln.

3 ICH Topic E 6 (R1) Guideline for Good Clinical Practice: www.emea.europa.eu/docs/en_GB/ document library/Scientific guideline/2009/09/ WC500002874.pdf

4 Verordnung über klinische Versuche mit Heilmitteln: www.admin.ch/ch/d/sr/c812_214_2.html

5 Bundesgesetz über Arzneimittel und Medizinprodukte (Heilmittelgesetz, HMG): www.admin.ch/ch/d/ sr/c812_21.html

6 Declaration of Helsinki: www.wma.net/en/20activities/ 10ethics/10helsinki/

7 Bundesgesetz über die Forschung am Menschen (Humanforschungsgesetz, HFG): www.bag.admin.ch/ themen/medizin/00701/00702/index.html?lang=de 